\title{
Drug Resistance Mechanisms in Tuberculosis
}

Created by: Lanfranco Fattorini , LAngelo lacobino , RFederico Giannoni Version received: 10 April 2020

The increased incidence of multidrug-resistant (MDR) Mycobacterium tuberculosis (Mtb) strains, defined as resistant to at least isoniazid and rifampin, the two highly bactericidal first-line drugs, is a major concern for tuberculosis (TB) control. The worldwide estimate of almost half a million incident cases of MDR/rifampin-resistant TB, is causing increasing concern. In this view, it is important to continuously update the knowledge on the mechanisms involved in the development of drug-resistant TB. Clinical, biological and microbiological reasons account for the generation of resistance, including: (i) nonadherence of patients to their therapy, and/or errors of physicians in therapy management, (ii) complexity and poor vascularization of granulomatous lesions, which obstruct drug distribution to some sites, resulting in resistance development, (iii) intrinsic drug resistance of tubercle bacilli, (iv) formation of non-replicating, drug-tolerant bacilli inside the granulomas, (v) development of mutations in Mtb genes, which are the most important molecular mechanisms of resistance. Here, a piece of information on the interplay of these factors is provided, to facilitate the clinical and microbiological management of drug-resistant TB at the global level, with attention also to the most recent diagnostic methods.

\section{Introduction}

Mycobacterium tuberculosis (Mtb) is the etiologic agent of tuberculosis (TB), the leading cause of death from a single infectious disease agent worldwide [1] ${ }^{[1]}$. In 2018, the World Health Organization (WHO) estimates of the global burden of TB were 10 million cases and 1.45 million deaths. The current antibiotic treatment of drug-susceptible TB requires administration of a combination therapy for 6 months, including the first-line drugs rifampin (RIF), isoniazid (INH), pyrazinamide (PZA) and ethambutol (EMB) for 2 months, followed by RIF and INH for 4 months.

For a number of reasons, development of drug-resistant Mtb strains may occur, including multidrug-resistant (MDR: resistant at least to INH and RIF) and extensively-drug-resistant (XDR) strains [MDR resistant to a fluoroquinolone (FQ) and a second-line injectable drug [kanamycin (KM), amikacin (AM), capreomycin (CM)] [2]]. WHO reported that in 2018 there were an estimated 484,000 incident cases of MDR/rifampin-resistant (RR) TB cases, including about 378,000 MDR-TB cases and 214,000 deaths. The average proportion of MDR-TB cases with XDR-TB was 6.2\% [1]]. At the global level, 3.4\% of new cases (patients never treated with anti-TB medicines, or treated for $<1$ month) and $18 \%$ of previously treated cases (patients treated for 1 month in the past) had MDR/RR-TB, with the highest proportion occurring in the former Soviet Union (FSU) countries. In the low incidence countries of the European Economic Area, the MDR-TB was more prevalent among migrants (particularly from the FSU) than the native population [ $\left.{ }^{[3][4]}\right]$.

\section{Drug Resistance Mechanisms}

If the 6-months combination therapy for the treatment of drug-susceptible TB is adequately taken, patients achieve cure rates of $>95 \%$. The resistance developed by Mtb to any antimicrobial agent is not due to a single mechanism, but to the interplay of biological, clinical and microbiological reasons, including

1. Nonadherence of patients to therapy and/or errors of physicians in the therapy management (human errors), that increases the risk of developing genetically drug-resistant bacilli [[]]]];

2. Complexity and poor vascularization of granulomatous lesions, which obstruct drug distribution to some sites, leading to suboptimal drug concentration and development of phenotypic and genetic resistance [ [7][8];

3. Naturally occurring high levels of antibiotic resistance in tubercle bacilli (intrinsic resistance) [e][10][11]];

4. Formation of non-replicating (NR) drug-tolerant bacilli inside the granulomas (phenotypic resistance) [12][13]];

5. Development of genetically resistant bacilli by chromosomal mutations (acquired resistance) [14][15][16]]. 


\section{Human Errors}

Human errors may contribute to development of drug-resistance. Two pathways lead to genetic resistance: (i) primary resistance, when a person is infected with a drug-resistant strain, and (ii) acquired resistance, when a person infected with a drug susceptible strain is inadequately treated with drugs, allowing the selection of resistant mutants [17]]. The first case mostly occurs in highly crowded communities (e.g., prisons), or in countries with high MDR-TB prevalence, where it is essential to rapidly diagnose and treat patients, so as to reduce transmission [6]]. In the second case, it is essential to follow the WHO recommendations on how to adequately treat the TB patients whose disease is caused by a drug-susceptible strain. The clinicians need also to ensure that infection control measures are established, particularly when MDR-patients are hospitalized.

WHO identified a number of factors contributing to poor treatment outcomes, including acquisition of acquired drugresistant TB [17]]. They were: (i) Inappropriate treatment by health care providers (inappropriate or absent guidelines, poor training of physicians and nurses, sub-optimal education of patients, poor management of adverse drug reactions, no monitoring of treatment, poorly organized or funded TB control programs); (ii) Inadequate drug supply (poor quality medicines, stock-outs, poor storage conditions, wrong dose or combination); (iii) Inadequate drug intake or treatment response by patients (lack of information on treatment adherence, adverse effects, malabsorption).

Common clinical errors in MDR-TB management, particularly in developing countries, include the addition of a single drug to a failing regimen, failure to recognize existing drug resistance, failure to provide directly observed therapy and to manage nonadherence, suboptimal dosages of second-line drugs to decrease side effects, drug treatment based on

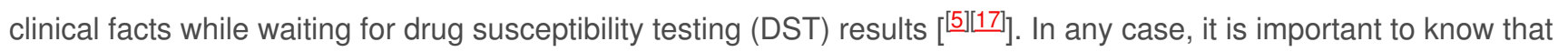
only drug combinations decrease the risk of selection of resistant strains.

\section{Complexity of TB Granulomas}

Long lasting therapies are also attributable to the complex pathology of TB. In the lungs of TB patients, a spectrum of heterogeneous granulomatous lesions coexist, ranging from well-vascularized cellular granulomas, in which a rim of lymphocytes surrounds macrophages and neutrophils, to avascular caseous granulomas, characterized by a necrotic center with a cheese-like aspect (caseum) formed by the lysis of host cells and bacteria [18][19]]. In these lesions, tubercle bacilli range from actively replicating (AR) stages, particularly in cellular granulomas, to dormant, slowlyreplicating or NR stages, typical of hypoxic caseous granulomas [이리]

The current 4-drugs therapeutic regimen (RIF-INH-PZA-EMB) is effective against AR intracellular bacilli in cellular granulomas, while NR extracellular bacilli localized in $\mathrm{pH}$-neutral, caseous granulomas are refractory to drug action [8][22][23][24] ]. The necrotic center of caseous granulomas contains NR bacilli phenotypically resistant to several drugs (drug-tolerant persisters), with the exception of rifamycins, which are known to sterilize caseum in ex-vivo assays [24][25]]. Spatial and temporal differences in drug distribution and the kinetics of accumulation of drugs in specific lesion compartments may create local windows of monotherapy that increase the risk of the emergence of drug-resistance []ㄹㄹㅡ] In this view, drug combinations should contain complementary drugs preferentially distributing in lesions in which their most vulnerable target population resides [[]]

In the event of caseous granulomas expansion, the necrotic centers fuse with the airway structures of bronchi to form pulmonary cavities in which are found both extracellular bacilli in liquefied caseum and intracellular bacilli derived from the lysis of infected macrophages of the cavity walls. In contact with the atmospheric oxygen, these bacilli rapidly proliferate in the lumen of cavities, and later appear in the sputum of TB patients [[8] ]. Due to high bacterial load in pulmonary cavities, genetically resistant bacilli with chromosomal mutations may be generated, playing an important role in the development of resistance [7]. Drug-specific gradients in the walls of human pulmonary cavities were reported to be associated with the development of acquired resistance in patients with MDR-TB, due to the low level of some drugs in the cavities centers, where there is a high number of replicating bacilli [2] ] $]$.

\section{Intrinsic Drug-Resistance}

During the evolution, Mtb developed mechanisms of intrinsic resistance involving cell envelope, efflux systems and 
other mechanisms (drug degradation and modification, target modification). Some examples of these mechanisms are provided in the following sections.

\section{Cell Envelope}

The constituents of the mycobacterial cell envelope are: the cytoplasmic membrane, the periplasmic space (PS), a network of peptidoglycan (PG), the arabinogalactan (AG), the long-chain mycolic acids (MA) and the capsule, made of a loose matrix of glucans and secreted proteins [ [28] ]. As to the first-line TB drugs, the bactericidal agent INH inhibits MA synthesis, while the bacteriostatic EMB inhibits AG synthesis and may sensitize Mtb to other drugs [료].

It is assumed that the innermost hydrophilic layers of PG and AG hinder the penetration of hydrophobic molecules. Instead, in the external part of the envelope, the hydrophobic MA layer formed by long-chain fatty acids restricts the penetration of hydrophilic drugs [[9][11]]. In principle, more lipophilic drugs, such as rifamycins, macrolides, and some FQs, diffuse by passive transport into and through the lipid-rich cell wall [29][30] $]$. However, the issue is perhaps more complex, since some studies showed that lipophilicity is an important but not exclusive factor of compound

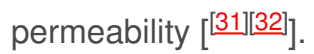

\section{Drug Efflux}

Efflux pumps (EPs) are transmembrane proteins that provide resistance by expelling the drugs from the interior to the exterior of the cell. Five EP families are known, organized on the basis of energetic and structural characteristics: the ATP-binding cassette (ABC) superfamily, the major facilitator superfamily (MFS), the multidrug and toxic compound extrusion (MATE) family, the small multidrug resistance (SMR) family and the resistance nodulation division (RND) superfamily [[9][10][30][33][34] . The ATP-energized ABC members are primary transporters, while the others are secondary transporters energized by proton gradients (MFS, SMR, RND) or sodium gradients (MATE). The EP of Mtb belongs to the ABC (representing 2.5\% of the entire Mtb genome), MFS and RND superfamilies, and to the SMR family.

Following exposure of Mtb to sub-inhibitory concentration of INH and EMB, EP genes are overexpressed resulting in the development of low-level resistance for a prolonged period of time. After several weeks, a high level of acquired resistance develops, caused by chromosomal mutations in the genes encoding the target proteins [[35][36] ]. These observations indicate that inappropriate TB treatment may generate pressure by sub-inhibitory drug concentrations that increase drug efflux, allowing a subsequent selection of mutants with high-level resistance [30][34]].

Several EPs are known to be associated with resistance. For instance, Mtb exposure to INH induces overexpression of $M m p L 7$ and $m m R$ EP genes [ [37][38] $]$. Furthermore, several EPs are involved in resistance to several drugs. Thus, the EP Tap mediates low-level resistance to tetracycline (TC) and aminoglycosides, whereas EPs encoded by the Rv0194 gene are associated with resistance to TC, $\beta$-lactams, streptomycin, chloramphenicol, and vancomycin. Mutations in the Rv0678 gene caused an up-regulation of the transport protein MmpL5, which caused EP-mediated cross-resistance to both bedaquiline (BDQ) and clofazimine (CFZ) [337][39][40]]. This is a potentially dangerous evolution of Mtb against antibiotics particularly in recent times, since BDQ and CFZ have just been included in the new WHO treatment guidelines of MDR/XDR-TB [441][42]].

\section{Other Mechanisms}

The most important mechanisms of the intrinsic drug resistance of Mtb are considered to be the lipid-rich cell wall and the EP, but other systems are known to neutralize toxic chemicals and antibiotics, including drug inactivation or modification, and target modification.

Among drug inactivating enzymes, Mtb $\beta$-lactamases, which are probably localized in the PS, are less effective than those of other bacteria to hydrolyse $\beta$-lactams, but their activity, together with slow penetration across the cell wall and low affinity for penicillin-binding proteins, is good enough to render Mtb intrinsically resistant to most $\beta$-lactams [[9][11]].

As to aminoglycosides (KM, AM) and cyclic peptides (CM), Mtb is able to inactivate them by acetylation performed by the enhanced intracellular survival protein encoded by eis, whose expression is upregulated by the MDR transcription 
regulator WhiB7 [11]]. Promoter mutations lead to an overexpression of eis, resulting in low-level resistance to KM, but not AM [[43]].

M. tuberculosisis naturally resistant to macrolides (e.g., clarithromycin and azithromycin) because of the inducible erm(37), a ribosomal RNA methyltransferase which alters ribosomes by methylating the $23 \mathrm{~S}$ rRNA [444][45]].

\section{Phenotypic Drug-Resistance}

Caseous granulomas and the cavities of the lungs of TB patients harbor subpopulations of NR bacilli which are phenotypically drug-resistant but genetically susceptible, commonly referred to as persisters. Characterized by a transient, non-heritable drug tolerance, persisters are capable of withstanding bactericidal drug concentrations, and once the antibiotic is removed, to resume growth with genetic features identical to the original strain.

The level of resistance to different antimicrobial agents varies with the in vitro stress model used[20][23][46][47][48][49]], including hypoxia (Wayne dormancy model) [ [50[51]], nutrient starvation [52]], acids and/or nitric oxide [53][54]], stationary phase [ $[55]$, antibiotic-starved strains $\left[{ }^{[56}\right]$ and others, or their variants. In the Wayne model, in which dormant bacilli are obtained by a gradual adaptation to anaerobiosis through the self-generated formation of an oxygen gradient, nonreplicating persistence (NRP) stages 1 and 2 were observed [[50]]. NRP-2 cells developed a thickened outer layer that helped in restricting RIF entry [ $\left.{ }^{[57}\right]$. Our group used the Wayne model at different pHs: $\mathrm{pH}$ 6.6, the $\mathrm{pH}$ or culture media [ [58]], pH 5.8, to mimic the environment of cellular granulomas [59], $\mathrm{pH} 7.3$, to mimic the environment of caseous granulomas [60]]. We found that at $\mathrm{pH} 5.8$, several drugs killed NR bacilli, with the best being the rifamycins RIF and rifapentine (RFP), while at $\mathrm{pH} 7.3$, only RIF and RFP killed dormant bacilli out of 12 drugs tested [60]]. Since the rifamycins were the only agents sterilizing caseum obtained from rabbits [24][25]], our model could mimic caseum to measure drug activity against NR Mtb in this environment. In hypoxia at pH 7.3, we found that RIF plus nitazoxanide (a nitro-compound for anaerobic infections) killed NR Mtb cells, while the combination currently used for human TB therapy (RIF-INH-PZA-EMB) did not [[61] . Dormancy is not necessary or sufficient for Mtb persistence, indicating that persistence is a phenomenon more complex than dormancy, and that additional characteristics are needed to define the persister phenotypes, which depends on the NR model used [[62]].

Two kinds of persisters are known [46]]: (i) Class I, rare, generated in a replicating population, formed continuously and in a purely stochastic manner. They are bacilli phenotypically tolerant to different antibiotics by different mechanisms, and it is likely that the overall population can be killed by drug combinations; (ii) Class II, abundant, involving almost all of the cells in a population, e.g., in the stationary phase, hypoxic conditions, nutrient starvation. Growth arrest is associated with resistance to a large number of drugs, and it is likely that new kinds of antibiotics are necessary to overcome these cells [466]].

Interestingly, RIF-resistant or moxifloxacin (MXF) resistant mutants carrying mutations inrpoB or gyrA genes emerged at high frequency from the persistent phase of Mtb cells exposed to RIF for prolonged periods. These cells carried elevated levels of the hydroxyl radical, which inflicted genome-wide mutations facilitating resistance to the same, or another, antibiotic [63][64][65]]. In consideration of the long TB therapy, these observations may have clinical significance in the emergence of drug-resistant mutants if local monotherapy occurs in patients who do not correctly take multi-drug TB therapy. In this view, it was postulated that persisters behave as an evolutionary reservoir from which drugresistant mutants can emerge [13] $]$.

\section{Acquired Drug-Resistance and drug susceptibility testing}

A cocktail of different drugs is used to treat TB. Each molecule binds to one or more target, thus inhibiting their functions. The continuous drug exposure during lengthy treatments and the noncompliance of patients to drug regimens, pushes Mtb to select for mutations in genes encoding drug targets, responsible for development of the majority of resistances in clinical strains [ [11]]. A list of the major target genes that, in the case of mutation, confer resistance to the drugs of the recently reported WHO treatment groups A, B and C [41]][42]], is shown in Table 1

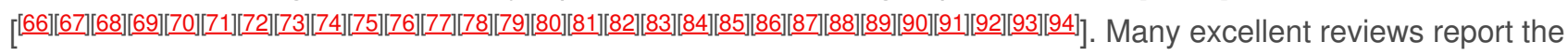
genetic mechanisms involved in this resistance to RIF, INH, KM, CM and other drugs [[9][14][15][67][87][95]]. 
Phenotypic testing is still considered a gold standard for Mtb DST, which is accurate, but takes at least two weeks for results [ [67]]. However, a pivotal role has been recently played by the more and more rapid molecular methods to diagnose drug-resistant TB by the identification of chromosomal mutations, including line probe assays, the Xpert MTB/RIF system (Cepheid, Sunnyvale, CA, USA), target gene sequencing, whole genome sequencing (WGS), point-

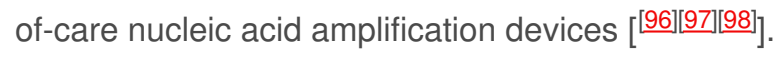

The Treatment Action Group (TAG) recently released the pipeline report 2019 on TB diagnostics [99]]. The TAGstratified DST tests for decentralized and centralized laboratories. As to the decentralized tests, the Xpert MTB/RIF assay (sensitivity and specificity for RIF resistance of $96 \%$ and $98 \%$, respectively) was recommended by the WHO in 2010 , and entered in the market in the same year. The sensitivity of this assay increased with the 2017 rollout of the Xpert MTB/RIF Ultra cartridge. In 2020, there is expected the WHO evaluation and market entry of Xpert XDR, which will detect resistance to INH, MFX, AM, KM, ofloxacin [일. In 2013, another company (Molbio Diagnostics, Goa, India) released its systems Truenat MTB and Truenat MTB-RIF Dx onto the Indian market [이] ]. In January 2020, a rapid WHO Communication reported that the Truenat systems MTB, MTB Plus and MTB RIF Dx assays showed comparable accuracy with Xpert MTB/RIF and Xpert Ultra for Mtb detection (Truenat MTB and Truenat MTB Plus), and for sequential RIF resistance detection (Truenat MTB RIF Dx) [1100]]. Furthermore, the data for Truenat MTB RIF Dx showed similar accuracy to the WHO approved commercial line probe assays indicated by the TAG for centralized DST [GenoType MTBDRplus Version 2.0 (Hain Lifescience, Nehren, Germany) and Nipro NTM+MDRTB detection kit2 (Nipro, Osaka, Japan)] [ㅂ9][100]]. Other systems marketed in 2015-2019 and on the pathway to the WHO evaluation for the centralized determination of molecular resistance to INH and RIF are: Cobas MTB-RIF/INH (Roche, Basel, Switzerland), BD MAX MDR-TB (Becton Dickinson, Sparks, MA, USA), real-time MTB-RIF/INH Resistance assay (Abbott, Abbott Park, IL, USA) and FluoroType MTBDR version 2.0 (RIF, INH) (Hain Lifescience) [[99][101][102][103].

Finally, the WGS technology is capable of identifying the complete drug-resistance profile of an Mtb strain, ideally enabling clinicians to obtain the best anti-TB treatment [67][98][100][104]]. However, more data are still needed to correlate genetic mutations with phenotypic resistance, in order to definitely guide the clinical care.

In this view, the initiative of the Comprehensive Resistance Prediction for Tuberculosis: an International Consortium (CRyPTIC) project aims at understanding the relationship between genotypes and resistance by sequencing 100,000 whole TB genomes from various countries, in parallel with comprehensive DST assays. Overall, at this stage, the WGS still needs more studies, but it is commonly believed that this technology will be the future of rapid, centralized DST $[09][105]$.

Table 1. Drugs of the World Health Organization (WHO) groups A, B and C, and list of the most common drug resistance-related target genes.

\begin{tabular}{|c|c|c|c|c|}
\hline Group & Drug* & $\begin{array}{l}\text { Target } \\
\text { Gene/s }\end{array}$ & Gene Product (Function Affected) & References \\
\hline \multirow[t]{4}{*}{ A } & $\begin{array}{l}\text { LFX or } \\
\text { MFX }\end{array}$ & gyrA & $\begin{array}{l}\text { DNA gyrase, subunit A (DNA } \\
\text { replication) }\end{array}$ & [66][67] \\
\hline & & gyrB & $\begin{array}{l}\text { DNA gyrase, subunit B (DNA } \\
\text { replication) }\end{array}$ & [68][69] \\
\hline & $\mathrm{BDQ}$ & atpE & $\begin{array}{l}\text { ATP synthase, subunit F0 (ATP } \\
\text { synthesis) }\end{array}$ & [69][0] \\
\hline & & $r v 0678$ & Transcriptional regulator (drug efflux) & [69][0] \\
\hline
\end{tabular}




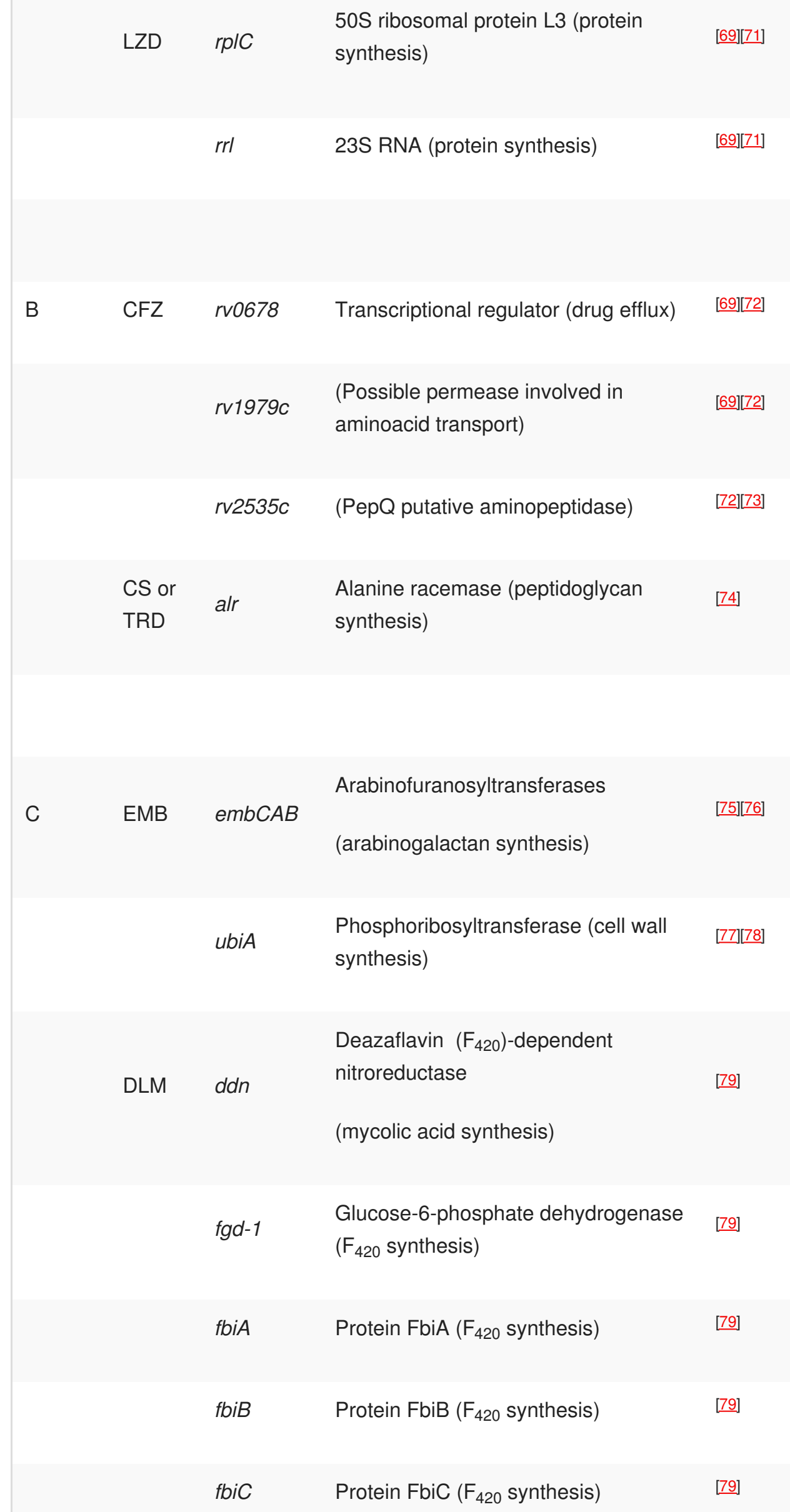


Pyrazinamidase (conversion of pro-

PZA pncA drug PZA into pyrazinoic acid, [67][80] resulting in dysfunctions of membrane potential)

rpsA $30 \mathrm{~S}$ ribosomal protein S1 (m-RNA trans-translation)

[14][80]

Aspartate decarboxylate

(panthotenate synthesis)

[80][81]

pand

$$
\text { (panthotenate synthesis) }
$$

clpc1 ATP-dependent ATP-ase (protein degradation)

[14][82]

IPM-

CLN

or

rv2518c

LdtB, nonclassical, L,D-

or transpeptidase (peptidoglycan

[10][83][84]

MPM synthesis)

rv3682

PonA2, penicillin-binding protein (peptidoglycan synthesis)

[10][83]

Rv2068c

blaC (major b-lactamase)

[85]

16S ribosomal RNA (protein synthesis)

[67][86]

AM rrs (a)

ribosomal protein S12 (protein synthesis)

[67][87]

SM rpsl synthesis)

16S ribosomal RNA (protein synthesis)

[67][86]

rrs

\begin{tabular}{|c|c|c|}
\hline rrs & $\begin{array}{l}\text { 16S ribosomal RNA (protein } \\
\text { synthesis) }\end{array}$ & [67][86] \\
\hline $\operatorname{gid} B$ & $\begin{array}{l}\text { (putative 16S rRNA } \\
\text { methyltransferase) }\end{array}$ & [88][89] \\
\hline$r v 0565 c$ & $\begin{array}{l}\text { Monoxygenase (activation of pro- } \\
\text { drugs ETO and PTO) }\end{array}$ & [90] \\
\hline ethA & $\begin{array}{l}\text { Monooxygenase (activation of pro- } \\
\text { drugs ETO and PTO) }\end{array}$ & [10][91] \\
\hline
\end{tabular}




\begin{tabular}{|c|c|c|c|}
\hline & mymA & $\begin{array}{l}\text { Monooxygenase (activation of pro- } \\
\text { drugs ETO and PTO) }\end{array}$ & [90][92] \\
\hline & katG & $\begin{array}{l}\text { Catalase-peroxidase (activation of } \\
\text { pro-drugs ETO, PTO, INH) }\end{array}$ & [91] \\
\hline & $\operatorname{inh} A$ & $\begin{array}{l}\text { Enoyl-ACP reductase (mycolic acid } \\
\text { synthesis) }\end{array}$ & [67][91] \\
\hline \multirow[t]{3}{*}{ PAS } & thy $A$ & Thymidylate synthase & [14][93] \\
\hline & folc & Dihydropholate synthase & [14][94] \\
\hline & $d f r A$ & Dihydropholate reductase & [14][94] \\
\hline
\end{tabular}

$\left(^{*}\right)$ LFX, levofloxacin; MFX, moxifloxacin; BDQ, bedaquiline; LZD, linezolid; CFZ, clofazimine; CS, cycloserine; TRD, terizidone; EMB, ethambutol; DLM, delamanid; PZA, pyrazinamide; IPM-CLN, imipenem-cilastatin; MPM, meropenem; AM, amikacin; SM, streptomycin; ETO, ethionamide; PTO, protionamide; PAS, para-aminosalicylic acid.

\section{References}

1. World Health Organization. Global Tuberculosis Report 2019; World Health Organization: Geneva, Switzerland, 2019.

2. Payam Nahid; Sundari R. Mase; Giovanni Battista Migliori; Giovanni Sotgiu; Graham H. Bothamley; Jan L. Brozek; Adithya Cattamanchi; J. Peter Cegielski; Lisa Chen; Charles L. Daley; et al.Tracy L. DaltonRaquel DuarteFederica FregoneseC. Robert HorsburghFaiz Ahmad KhanFayez KheirZhiyi LanAlfred LardizabalMichael LauzardoJoan M. ManganSuzanne M. MarksLindsay McKennaDick MenziesCarole D. MitnickDiana M. NilsenFarah ParvezCharles A. PeloquinAnn RafteryH. Simon SchaafNeha S. ShahJeffrey R. StarkeJohn W. WilsonJonathan M. WorthamTerence ChorbaBarbara Seaworth Treatment of Drug-Resistant Tuberculosis. An Official ATS/CDC/ERS/IDSA Clinical Practice Guideline. American Journal of Respiratory and Critical Care Medicine 2019, 200, e93-e142, 10.1164/rccm.201909-1874st.

3. Mustazzolu, A.; Borroni, E.; Cirillo, D.M.; Giannoni, F.; lacobino, A.; Italian Multicentre Study on Resistance to Antituberculosis Drugs (SMIRA); Fattorini, L. Trend in rifampicin-, multidrug- and extensively drug-resistant tuberculosis in Italy, 2009-2016. Eur. Respir. J. 2018, 52.

4. Bernard, C.; Brossier, F.; Sougakoff, W.; Veziris, N.; Frechet-Jachym, M.; Metivier, N.; Renvoisé, A.; Robert, J.; Jarlier, V. MDR-TB Management group of the NRC. A surge of MDR and XDR tuberculosis in France among patients born in the Former Soviet Union. Euro Surveill. 2013, 18.

5. Ignacio Monedero; José A Caminero; Common errors in multidrug-resistant tuberculosis management. Expert Review of Respiratory Medicine 2013, 8, 15-23, 10.1586/17476348.2014.856758.

6. Simon Tiberi; Emanuele Pontali; Marina Tadolini; Lia D'Ambrosio; Giovanni Sotgiu; Challenging MDR-TB clinical problems - The case for a new Global TB Consilium supporting the compassionate use of new anti-TB drugs. International Journal of Infectious Diseases 2019, 80, S68-S72, 10.1016/j.jiji.2019.01.040.

7. Natasha Strydom; Sneha V. Gupta; William S. Fox; Laura E. Via; Hyeeun Bang; Myungsun Lee; Seokyong Eum; Tae Sun Shim; Clifton E. Barry; Matthew Zimmerman; Véronique Dartois; Rada M. Savic; Tuberculosis drugs' distribution and emergence of resistance in patient's lung lesions: A mechanistic model and tool for regimen and dose optimization.. PLoS Medicine 2019, 16, e1002773, 10.1371/journal.pmed.1002773.

8. Véronique Dartois; The path of anti-tuberculosis drugs: from blood to lesions to mycobacterial cells. Nat. Rev. Microbiol. 2014, 12, 159-167, 10.1038/nrmicro3200.

9. Richa Singh; Surya Prakash Dwivedi; Usha Singh Gaharwar; Ramovatar Meena; Paulraj Rajamani; Tulika Prasad; Recent updates on drug resistance in Mycobacterium tuberculosis. Journal of Applied Microbiology 2019, null, , 10.1111/jam.14478.

10. Mohammad Javad Nasiri; Mehri Haeili; Mona Ghazi; Hossein Goudarzi; Ali Pormohammad; Abbas Ali Imani Fooladi; Mohammad M. Feizabadi; New Insights in to the Intrinsic and Acquired Drug Resistance Mechanisms in Mycobacteria. Frontiers in Microbiology 2017, 8, 1529, 10.3389/fmicb.2017.00681. 
11. Nguyen, L.; Antibiotic resistance mechanisms in M. tuberculosis: An update. Arch. Toxicol. 2016, 90, 1585-1604.

12. Brauner, A.; Fridman, O.; Gefen, O.; Balaban, N.Q.; Distinguishing between resistance, tolerance and persistence to antibiotic treatment. Nat. Rev. Microbiol. 2016, 14, 320-330.

13. Nadia R. Cohen; Michael A. Lobritz; James J. Collins; Microbial persistence and the road to drug resistance.. Cell Host \& Microbe 2013, 13, 632-42, 10.1016/j.chom.2013.05.009.

14. H. M. Adnan Hameed; Mahmudul Islam; Chiranjibi Chhotaray; Changwei Wang; Yang Liu; Yaoju Tan; Xinjie Li; Shouyong Tan; Vincent Delorme; Wing W. Yew; Jianxiong Liu; Tianyu Zhang; Molecular Targets Related Drug Resistance Mechanisms in MDR-, XDR-, and TDR-Mycobacterium tuberculosis Strains. Frontiers in Microbiology 2018, 8, 114, 10.3389/fcimb.2018.00114.

15. Vahid Lohrasbi; Malihe Talebi; Abed Zahedi Bialvaei; Lanfranco Fattorini; Michel Drancourt; Mohsen Heidary; Davood DarbanSarokhalil; Trends in the discovery of new drugs for Mycobacterium tuberculosis therapy with a glance at resistance. Tuberculosis 2018, 109, 17-27, 10.1016/j.tube.2017.12.002.

16. Mahmudul Islam; H.M. Adnan Hameed; Julius Mugweru; Chiranjibi Chhotaray; Changwei Wang; Yaoju Tan; Jianxiong Liu; Xinjie Li; Shouyong Tan; Iwao Ojima; Wing Wai Yew; Eric Nuermberger; Gyanu Lamichhane; Tianyu Zhang; Drug resistance mechanisms and novel drug targets for tuberculosis therapy. Journal of Genetics and Genomics 2017, 44, 21-37, 10.1016/j.jgg.2016.10.002.

17. World Health Organization. Companion Handbook to the WHO Guidelines for the Programmatic Management of Drug-Resistant Tuberculosis; World Health Organization: Geneva, Switzerland, 2014.

18. Clifton E. Barry; Helena I. Boshoff; Véronique Dartois; Thomas Dick; Sabine Ehrt; Joanne Flynn; Dirk Schnappinger; Robert J Wilkinson; Douglas Young; The spectrum of latent tuberculosis: rethinking the biology and intervention strategies. Nat. Rev. Microbiol. 2009, 7, 845-855, 10.1038/nrmicro2236.

19. Anne Lenaerts; Clifton E. Barry; Véronique Dartois; Heterogeneity in tuberculosis pathology, microenvironments and therapeutic responses. Immunological Reviews 2015, 264, 288-307, 10.1111/imr.12252.

20. Angelo lacobino; Giovanni Piccaro; Federico Giannoni; Alessandro Mustazzolu; Lanfranco Fattorini; Fighting tuberculosis by drugs targeting nonreplicating Mycobacterium tuberculosis bacilli.. International Journal of Mycobacteriology 2017, 6, $213-221$.

21. Jansy P. Sarathy; Fabio Zuccotto; Ho Hsinpin; Lars Sandberg; Laura E. Via; Gwendolyn A. Marriner; Thierry Masquelin; Paul Wyatt; Peter C. Ray; Véronique Dartois; Prediction of Drug Penetration in Tuberculosis Lesions. ACS Infectious Diseases 2016, 2, 552-563, 10.1021/acsinfecdis.6b00051.

22. Jean-Philippe Lanoix; Anne J. Lenaerts; Eric L. Nuermberger; Heterogeneous disease progression and treatment response in a C3HeB/FeJ mouse model of tuberculosis.. Disease Models \& Mechanisms 2015, 8, 603-10, 10.1242/dmm.019513.

23. G. Piccaro; Perla Filippini; F. Giannoni; Luigi Scipione; Silvano Tortorella; D. De Vita; Paolo Mellini; Lanfranco Fattorini; Activity of Drugs Against DormantMycobacterium tuberculosis. Journal of Chemotherapy 2011, 23, 175-178, 10.1179/joc.2011.23.3.175.

24. Jansy P. Sarathy; Hsin-Pin Ho Liang; Danielle Weiner; Jacqueline Gonzales; Laura E. Via; Véronique Dartois; An In Vitro Caseum Binding Assay that Predicts Drug Penetration in Tuberculosis Lesions. Journal of Visualized Experiments 2017, null, , $10.3791 / 55559$.

25. Jansy P. Sarathy; Laura E. Via; Danielle Weiner; Landry Blanc; Helena Boshoff; Eliseo A. Eugenin; Clifton E. Barry; Véronique Dartois; Extreme Drug Tolerance of Mycobacterium tuberculosis in Caseum. Antimicrobial Agents and Chemotherapy 2017, 62, e02266-17, 10.1128/aac.02266-17.

26. Prideaux, B.; Via, L.E.; Zimmerman, M.D.; Eum, S.; Sarathy, J.; O’Brien, P.; Chen, C.; Kaya, F.; Weiner, D.M.; Chen, P.Y.; et al.et al. The association between sterilizing activity and drug distribution into tuberculosis lesions. Nat. Med. 2015, 21, 1223-1227.

27. Keertan Dheda; Laura Lenders; Gesham Magombedze; Shashikant Srivastava; Prithvi Raj; Erland Arning; Paula Ashcraft; Teodoro Bottiglieri; Helen Wainwright; Timothy Pennel; et al.Anthony LinegarLoven MoodleyAnil PooranJotam G. PasipanodyaFrederick A. SirgelPaul D. Van HeldenEdward WakelandR. M. WarrenTawanda Gumbo Drug-Penetration Gradients Associated with Acquired Drug Resistance in Patients with Tuberculosis. American Journal of Respiratory and Critical Care Medicine 2018, 198, 1208-1219, 10.1164/rccm.201711-2333oc.

28. Charles L. Dulberger; Eric J. Rubin; Cara C. Boutte; The mycobacterial cell envelope - a moving target.. Nat. Rev. Microbiol. 2019, 18, 47-59, 10.1038/s41579-019-0273-7.

29. Jansy Passiflora Sarathy; Véronique Dartois; Edmund Jon Deoon Lee; The Role of Transport Mechanisms in Mycobacterium Tuberculosis Drug Resistance and Tolerance. Pharmaceuticals 2012, 5, 1210-1235, 10.3390/ph5111210.

30. Diana Machado; Miriam Girardini; Miguel Viveiros; Marco Pieroni; Challenging the Drug-Likeness Dogma for New Drug Discovery in Tuberculosis. Frontiers in Microbiology 2018, 9, , 10.3389/fmicb.2018.01367.

31. Sebastian M. Gygli; Sonia Borrell; Andrej Trauner; Sebastien Gagneux; Antimicrobial resistance in Mycobacterium tuberculosis: mechanistic and evolutionary perspectives. FEMS Microbiology Reviews 2017, 41, 354-373, 10.1093/femsre/fux011.

32. Sridhara Janardhan; M. Ram Vivek; G. Narahari Sastry; Modeling the permeability of drug-like molecules through the cell wall of Mycobacterium tuberculosis: an analogue based approach. Molecular BioSystems 2016, 12, 3377-3384, 10.1039/C6MB00457A.

33. Edda De Rossi; José A. Aínsa; Giovanna Riccardi; Josã@ A. Aãnsa; Role of mycobacterial efflux transporters in drug resistance: an unresolved question. FEMS Microbiology Reviews 2006, 30, 36-52, 10.1111/j.1574-6976.2005.00002.x.

34. Liliana Rodrigues; Tanya Parish; Meenakshi Balganesh; José A. Ainsa; José Antonio Aínsa Claver; Antituberculosis drugs: reducing efflux = increasing activity. Drug Discovery Today 2017, 22, 592-599, 10.1016/j.drudis.2017.01.002.

35. Shashikant Srivastava; Sandirai Musuka; Carleton Sherman; Claudia Meek; Richard Leff; Tawanda Gumbo; Efflux-pump-derived 
multiple drug resistance to ethambutol monotherapy in Mycobacterium tuberculosis and the pharmacokinetics and pharmacodynamics of ethambutol.. The Journal of Infectious Diseases 2010, 201, 1225-31, 10.1086/651377.

36. Diana Machado; Isabel Couto; João Perdigão; Liliana Rodrigues; Isabel Portugal; Pedro V. Baptista; Bruno Veigas; Leonard Amaral; Miguel Viveiros; Contribution of Efflux to the Emergence of Isoniazid and Multidrug Resistance in Mycobacterium tuberculosis. PLOS ONE 2012, 7, e34538, 10.1371/journal.pone.0034538.

37. Liliana Rodrigues; Cristina Villellas; Rebeca Bailo; Miguel Viveiros; José A. Aínsa; Role of the Mmr Efflux Pump in Drug Resistance in Mycobacterium tuberculosis. Antimicrobial Agents and Chemotherapy 2012, 57, 751-757, 10.1128/AAC.01482-12.

38. Maria Rosalia Pasca; Paola Guglierame; Edda De Rossi; Francesca Zara; Giovanna Riccardi; mmpL7 Gene of Mycobacterium tuberculosis Is Responsible for Isoniazid Efflux in Mycobacterium smegmatis. Antimicrobial Agents and Chemotherapy 2005, 49, 4775-4777, 10.1128/aac.49.11.4775-4777.2005.

39. Ruben Christiaan Hartkoorn; Swapna Uplekar; Stewart T. Cole; Cross-Resistance between Clofazimine and Bedaquiline through Upregulation of MmpL5 in Mycobacterium tuberculosis. Antimicrobial Agents and Chemotherapy 2014, 58, 2979-2981, 10.1128/AAC.00037-14.

40. Nabila Ismail; Remco P.H. Peters; Nazir A. Ismail; Shaheed V. Omar; Clofazimine Exposure In Vitro Selects Efflux Pump Mutants and Bedaquiline Resistance. Antimicrobial Agents and Chemotherapy 2019, 63, , 10.1128/aac.02141-18.

41. World Health Organization. WHO Consolidated Guidelines on Drug-Resistant Tuberculosis Treatment; World Health Organization: Geneva, Switzerland, 2019.

42. World Health Organization. Rapid Communication: Key Changes to the Treatment of Drug-Resistant Tuberculosis; World Health Organization: Geneva, Switzerland, 2019.

43. Sophia B. Georghiou; Marisa Magaña; Richard S. Garfein; Donald G. Catanzaro; Antonino Catanzaro; Timothy C. Rodwell; Evaluation of Genetic Mutations Associated with Mycobacterium tuberculosis Resistance to Amikacin, Kanamycin and Capreomycin: A Systematic Review. PLOS ONE 2012, 7, e33275, 10.1371/journal.pone.0033275.

44. Karolína Buriánková; Florence Doucet-Populaire; Olivier Dorson; Anne Gondran; Jean-Claude Ghnassia; Jaroslav Weiser; Jean-Luc Pernodet; Molecular Basis of Intrinsic Macrolide Resistance in the Mycobacterium tuberculosis Complex. Antimicrobial Agents and Chemotherapy 2004, 48, 143-150, 10.1128/aac.48.1.143-150.2004.

45. C. T. Madsen; L. Jakobsen; K. Buriánková; Florence Doucet-Populaire; Jean-Luc Pernodet; Stephen Douthwaite; Methyltransferase Erm(37) Slips on rRNA to Confer Atypical Resistance in Mycobacterium tuberculosis. Journal of Biological Chemistry 2005, 280, 38942-38947, 10.1074/jbc.m505727200.

46. Ben Gold; Carl Nathan; Targeting Phenotypically Tolerant Mycobacterium tuberculosis. Microbiology Spectrum 2017, 5, 317-360, 10.1128/microbiolspec.tbtb2-0031-2016.

47. Samuel Lipworth; R.J.H. Hammond; V.O. Baron; Yanmin. Hu; A. Coates; Stephen H Gillespie; Defining dormancy in mycobacterial disease. Tuberculosis 2016, 99, 131-142, 10.1016/j.tube.2016.05.006.

48. Robert J. H. Hammond; Vincent O. Baron; Samuel Lipworth; Stephen H Gillespie; Enhanced Methodologies for Detecting Phenotypic Resistance in Mycobacteria. Methods Mol. Biol. 2018, 1736, 85-94, 10.1007/978-1-4939-7638-6_8.

49. Batyrshina, Y.R.; Schwartz, Y.S. Modeling of Mycobacterium tuberculosis dormancy in bacterial cultures.Tuberculosis 2019, 117, 717.

50. Wayne, L.G.; Hayes, L.G. An in vitro model for sequential study of shiftdown of Mycobacterium tuberculosisthrough two stages of nonreplicating persistence. Infect. Immun. 1996, 64, 2062-2069.

51. Charles D. Sohaskey; Martin I. Voskuil; In Vitro Models That Utilize Hypoxia to Induce Non-replicating Persistence in Mycobacteria. Methods Mol. Biol. 2015, 1285, 201-213, 10.1007/978-1-4939-2450-9_11.

52. Joanna Betts; Pauline T. Lukey; Linda C. Robb; Ruth A. McAdam; Ken Duncan; Evaluation of a nutrient starvation model ofMycobacterium tuberculosispersistence by gene and protein expression profiling. Molecular Microbiology 2002, 43, 717-731, 10.1046/j.1365-2958.2002.02779.x.

53. Martin I. Voskuil; Dirk Schnappinger; Kevin C. Visconti; Maria I. Harrell; Gregory M. Dolganov; David R. Sherman; Gary K. Schoolnik; Inhibition of Respiration by Nitric Oxide Induces a Mycobacterium tuberculosis Dormancy Program. Journal of Experimental Medicine 2003, 198, 705-713, 10.1084/jem.20030205.

54. Luiz Pedro S. De Carvalho; Gang Lin; Xiuju Jiang; Carl Nathan; Nitazoxanide Kills Replicating and NonreplicatingMycobacterium tuberculosisand Evades Resistance. Journal of Medicinal Chemistry 2009, 52, 5789-5792, 10.1021/jm9010719.

55. Yanmin Hu; Joseph A. Mangan; Jasvir Dhillon; Kath M. Sole; Denis A. Mitchison; P.D. Butcher; Anthony R. M. Coates; Detection of mRNA Transcripts and Active Transcription in Persistent Mycobacterium tuberculosisInduced by Exposure to Rifampin or Pyrazinamide. Journal of Bacteriology 2000, 182, 6358-6365, 10.1128/jb.182.22.6358-6365.2000.

56. Claudia Sala; Neeraj Dhar; Ruben Christiaan Hartkoorn; Ming Zhang; Young Hwan Ha; Patricia Schneider; Stewart T. Cole; Simple Model for Testing Drugs against Nonreplicating Mycobacterium tuberculosis $\nabla$ †. Antimicrobial Agents and Chemotherapy 2010, 54, 4150-4158, 10.1128/AAC.00821-10.

57. Kishor Jakkala; Parthasarathi Ajitkumar; Hypoxic Non-replicating Persistent Mycobacterium tuberculosis Develops Thickened Outer Layer That Helps in Restricting Rifampicin Entry.. Frontiers in Microbiology 2019, 10, 2339, 10.3389/fmicb.2019.02339.

58. Perla Filippini; Elisabetta Iona; Giovanni Piccaro; Pascale Peyron; Olivier Neyrolles; Lanfranco Fattorini; Activity of Drug Combinations against Dormant Mycobacterium tuberculosis. Antimicrobial Agents and Chemotherapy 2010, 54, 2712-2715, 
10.1128/aac.01736-09.

59. Giovanni Piccaro; Federico Giannoni; Perla Filippini; Alessandro Mustazzolu; Lanfranco Fattorini; Activities of Drug Combinations against Mycobacterium tuberculosis Grown in Aerobic and Hypoxic Acidic Conditions. Antimicrobial Agents and Chemotherapy 2013, 57, 1428-1433, 10.1128/AAC.02154-12.

60. Angelo lacobino; Giovanni Piccaro; Federico Giannoni; Alessandro Mustazzolu; Lanfranco Fattorini; Mycobacterium tuberculosis Is Selectively Killed by Rifampin and Rifapentine in Hypoxia at Neutral pH. Antimicrobial Agents and Chemotherapy 2016, 61, null-16, 10.1128/aac.02296-16.

61. Angelo lacobino; Federico Giannoni; Manuela Pardini; Giovanni Piccaro; Lanfranco Fattorini; The Combination RifampinNitazoxanide, but Not Rifampin-Isoniazid-Pyrazinamide-Ethambutol, Kills Dormant Mycobacterium tuberculosis in Hypoxia at Neutral pH.. Antimicrobial Agents and Chemotherapy 2019, 63, no, 10.1128/AAC.00273-19.

62. Mehmet A. Orman; Mark P. Brynildsen; Dormancy Is Not Necessary or Sufficient for Bacterial Persistence. Antimicrobial Agents and Chemotherapy 2013, 57, 3230-3239, 10.1128/AAC.00243-13.

63. Jees Sebastian; Sharmada Swaminath; Rashmi Ravindran Nair; Kishor Jakkala; Atul Pradhan; Parthasarathi Ajitkumar; De Novo Emergence of Genetically Resistant Mutants of Mycobacterium tuberculosis from the Persistence Phase Cells Formed against Antituberculosis Drugs In Vitro. Antimicrobial Agents and Chemotherapy 2016, 61, 16, 10.1128/aac.01343-16.

64. Rashmi Ravindran Nair; Deepti Sharan; Jees Sebastian; Sharmada Swaminath; Parthasarathi Ajitkumar; Heterogeneity of ROS levels in antibiotic-exposed mycobacterial subpopulations confers differential susceptibility.. Microbiology 2019, 165, 668-682, 10.1099/mic.0.000797.

65. Giovanni Piccaro; Donatella Pietraforte; Federico Giannoni; Alessandro Mustazzolu; Lanfranco Fattorini; Rifampin Induces Hydroxyl Radical Formation in Mycobacterium tuberculosis. Antimicrobial Agents and Chemotherapy 2014, 58, 7527-7533,

10.1128/AAC.03169-14.

66. Yu Pang; Zhaojing Zong; Fengmin Huo; Wei Jing; Yifeng Ma; Lingling Dong; Yunxu Li; Liping Zhao; Yuhong Fu; Hairong Huang; In Vitro Drug Susceptibility of Bedaquiline, Delamanid, Linezolid, Clofazimine, Moxifloxacin, and Gatifloxacin against Extensively DrugResistant Tuberculosis in Beijing, China. Antimicrobial Agents and Chemotherapy 2017, 61, e00900-17, 10.1128/aac.00900-17.

67. Paolo Miotto; Belay Tessema; Elisa Tagliani; Leonid Chindelevitch; Angela M. Starks; Claudia Emerson; Debra Hanna; Peter S. Kim; Richard Liwski; Matteo Zignol; Christopher Gilpin; Stefan Niemann; Claudia M. Denkinger; Joy Fleming; R. M. Warren; Derrick Crook; James Posey; Sébastien Gagneux; Sven Hoffner; Camilla Rodrigues; Iñaki Comas; David M. Engelthaler; Megan Murray; David Alland; Leen Rigouts; C. Lange; Keertan Dheda; Rumina Hasan; Uma Devi K. Ranganathan; Ruth McNerney; Matthew Ezewudo; Daniela M. Cirillo; Marco Schito; Claudio Köser; Timothy C. Rodwell; A standardised method for interpreting the association between mutations and phenotypic drug resistance inMycobacterium tuberculosis. European Respiratory Journal $\mathbf{2 0 1 7}$ 50, 1701354, 10.1183/13993003.01354-2017.

68. Mahmood, N.; Abbas, S.N.; Faraz, N.; Shahid, S. Mutational analysis of gyrB at amino acids: G481A \& D505A in multidrug resistant (MDR) tuberculosis patients. J. Infect. Public Health. 2019, 12, 496-501.

69. N. Ismail; S.V. Omar; Remco P.H. Peters; N.A. Ismail; Collated data of mutation frequencies and associated genetic variants of bedaquiline, clofazimine and linezolid resistance in Mycobacterium tuberculosis. Data in Brief 2018, 20, 1975-1983,

10.1016/j.dib.2018.09.057.

70. Ismail, N.; Ismail, N.A.; Omar, S.V.; Peters, R.P.H. In Vitro Study of Stepwise Acquisition of rv0678 and atpE Mutations Conferring Bedaquiline Resistance. Antimicrob. Agents Chemother. 2019, 63.

71. Rui Pi; Qingyun Liu; Qi Jiang; Qian Gao; Characterization of linezolid-resistance-associated mutations in Mycobacterium tuberculosis through WGS.. Journal of Antimicrobial Chemotherapy 2019, 74, 1795-1798, 10.1093/jac/dkz150.

72. Shuo Zhang; Jiazhen Chen; Peng Cui; Wanliang Shi; Wenhong Zhang; Ying Zhang; Identification of novel mutations associated with clofazimine resistance in Mycobacterium tuberculosis.. Journal of Antimicrobial Chemotherapy 2015, 70, 2507-10,

10.1093/jac/dkv150.

73. Deepak Almeida; Thomas loerger; Sandeep Tyagi; Si-Yang Li; Khisimuzi Mdluli; Koen Andries; Jacques Grosset; Jim Sacchettini; Eric Nuermberger; Mutations in pepQ Confer Low-Level Resistance to Bedaquiline and Clofazimine in Mycobacterium tuberculosis. Antimicrobial Agents and Chemotherapy 2016, 60, 4590-4599, 10.1128/AAC.00753-16.

74. Dimitrios Evangelopoulos; Gareth A. Prosser; Angela Rodgers; Belinda M. Dagg; Bhagwati Khatri; Mei Mei Ho; Maximiliano G. Gutierrez; Teresa Cortes; Luiz Pedro S. De Carvalho; Comparative fitness analysis of D-cycloserine resistant mutants reveals both fitness-neutral and high-fitness cost genotypes. Nature Communications 2019, 10, 4177-11, 10.1038/s41467-019-12074-z.

75. Sun, Q.; Xiao, T.Y.; Liu, H.C.; Zhao, X.Q.; Liu, Z.G.; Li, Y.N.; Zeng, H.; Zhao, L.L.; Wan, K.L. Mutations within embCAB Are Associated with Variable Level of Ethambutol Resistance in Mycobacterium tuberculosis Isolates from China. Antimicrob. Agents Chemother. 2017, 62.

76. Xinchang Chen; Guiqing He; Shiyong Wang; Siran Lin; Jia-Zhen Chen; Wenhong Zhang; Evaluation of Whole-Genome Sequence Method to Diagnose Resistance of 13 Anti-tuberculosis Drugs and Characterize Resistance Genes in Clinical Multi-Drug Resistance Mycobacterium tuberculosis Isolates From China.. Frontiers in Microbiology 2019, 10, 1741, 10.3389/fmicb.2019.01741.

77. Subramanya Lingaraju; Leen Rigouts; Aditi Gupta; Jongseok Lee; Alaine Nyaruhirira Umubyeyi; Amy Davidow; Susan German; Eunjin Cho; Ji-Im Lee; Sang-Nae Cho; Cheon Tae Kim; David Alland; Hassan Safi; Geographic Differences in the Contribution of ubiA Mutations to High-Level Ethambutol Resistance in Mycobacterium tuberculosis. Antimicrobial Agents and Chemotherapy 2016, 
60, 4101-4105, 10.1128/AAC.03002-15.

78. He, L.; Wang, X.; Cui, P.; Jin, J.; Chen, J.; Zhang, W.; Zhang, Y. ubiA (Rv3806c) encoding DPPR synthase involved in cell wall synthesis is associated with ethambutol resistance in Mycobacterium tuberculosis. Tuberculosis 2015, 95, 149-154.

79. Mamoru Fujiwara; Masanori Kawasaki; Norimitsu Hariguchi; Yongge Liu; Makoto Matsumoto; Mechanisms of resistance to delamanid, a drug for Mycobacterium tuberculosis. Tuberculosis 2017, 108, 186-194, 10.1016/j.tube.2017.12.006.

80. Ameeruddin Nusrath Unissa; Luke Elizabeth Hanna; Molecular mechanisms of action, resistance, detection to the first-line anti tuberculosis drugs: Rifampicin and pyrazinamide in the post whole genome sequencing era. Tuberculosis 2017, 105, 96-107, 10.1016/j.tube.2017.04.008.

81. Shi, W.; Chen, J.; Zhang, S.; Zhang, W.; Zhang, Y. Identification of Novel Mutations in LprG (rv1411c), rv0521, rv3630, rv0010c, ppsC and cyp128 Associated with Pyrazinoic Acid/Pyrazinamide Resistance inMycobacterium tuberculosis. Antimicrob. Agents Chemother. 2018, 62.

82. Shuo Zhang; Jiazhen Chen; Wanliang Shi; Peng Cui; Jia Zhang; Sanghyun Cho; Wenhong Zhang; Ying Zhang; Mutation in clpC1 encoding an ATP-dependent ATPase involved in protein degradation is associated with pyrazinamide resistance in Mycobacterium tuberculosis.. Emerging Microbes \& Infections 2017, 6, e8-2, 10.1038/emi.2017.1.

83. Shichun Lun; David Miranda; Andre Kubler; Haidan Guo; Mariama C. Maiga; Kathryn Winglee; Shaaretha Pelly; William R. Bishai; Synthetic Lethality Reveals Mechanisms of Mycobacterium tuberculosis Resistance to $\beta$-Lactams. mBio 2014, 5, e01767-14-14, 10.1128/mBio.01767-14.

84. Keira A. Cohen; Tal El-Hay; Kelly L. Wyres; Omer Weissbrod; Vanisha Munsamy; Chen Yanover; Ranit Aharonov; Oded Shaham; Thomas C. Conway; Yaara Goldschmidt; William R. Bishai; Alexander S Pym; Paradoxical Hypersusceptibility of Drug-resistant Mycobacteriumtuberculosis to $\beta$-lactam Antibiotics.. EBioMedicine 2016, 9, 170-179, 10.1016/j.ebiom.2016.05.041.

85. Laura Iannazzo; Daria Soroka; Sébastien Triboulet; Matthieu Fonvielle; Fabrice Compain; Vincent Dubée; Jean-Luc Mainardi; JeanEmmanuel Hugonnet; Emmanuelle Braud; Michel Arthur; Mélanie Etheve-Quelquejeu; Routes of Synthesis of Carbapenems for Optimizing Both the Inactivation of I,d-Transpeptidase LdtMt1 of Mycobacterium tuberculosis and the Stability toward Hydrolysis by B-Lactamase BlaC. Journal of Medicinal Chemistry 2016, 59, 3427-3438, 10.1021/acs.jmedchem.6b00096.

86. Vangala Ramakrishna; Pravin Kumar Singh; Shantanu Prakash; Amita Jain; Second Line Injectable Drug Resistance and Associated Genetic Mutations in Newly Diagnosed Cases of Multidrug-Resistant Tuberculosis.. Microbial Drug Resistance 2020, null, , 10.1089/mdr.2019.0215.

87. João Perdigão; Isabel Portugal; Genetics and roadblocks of drug resistant tuberculosis. Infection, Genetics and Evolution 2019, 72 , 113-130, 10.1016/j.meegid.2018.09.023.

88. Jitender S. Verma; Yash Gupta; Deepthi Nair; Nikhat Manzoor; Rajinder S. Rautela; Arvind Rai; Vishwa M. Katoch; Evaluation of gidB alterations responsible for streptomycin resistance in Mycobacterium tuberculosis. Journal of Antimicrobial Chemotherapy 2014, 69, 2935-2941, 10.1093/jac/dku273.

89. Sharon Y. Wong; Jong Seok Lee; Hyun Kyung Kwak; Laura E. Via; Helena I. M. Boshoff; Clifton E. Barry; Mutations in gidB Confer Low-Level Streptomycin Resistance in Mycobacterium tuberculosis $\nabla \dagger$. Antimicrobial Agents and Chemotherapy 2011, 55, 25152522, 10.1128/AAC.01814-10.

90. Nathan D. Hicks; Allison F. Carey; Jian Yang; Yanlin Zhao; Sarah M Fortune; Bacterial Genome-Wide Association Identifies Novel Factors That Contribute to Ethionamide and Prothionamide Susceptibility in Mycobacterium tuberculosis. mBio 2019, 10, e00616-19, 10.1128/mBio.00616-19.

91. Johana Rueda; Teresa Realpe; Gloria Isabel Mejia; Elsa Zapata; Juan Carlos Rozo; Beatriz Ferro; Jaime Robledo; Genotypic Analysis of Genes Associated with Independent Resistance and Cross-Resistance to Isoniazid and Ethionamide in Mycobacterium tuberculosis Clinical Isolates. Antimicrobial Agents and Chemotherapy 2015, 59, 7805-7810, 10.1128/AAC.01028-15.

92. Sarah Schmidt Grant; Samantha Wellington; Tomohiko Kawate; Christopher A. Desjardins; Melanie Silvis; Carl Wivagg; Matthew Thompson; Katherine Gordon; Edward Kazyanskaya; Raymond Nietupski; Nathan Haseley; Noriaki Iwase; Ashlee M. Earl; Michael Fitzgerald; Deborah T. Hung; Baeyer-Villiger Monooxygenases EthA and MymA Are Required for Activation of Replicating and Nonreplicating Mycobacterium tuberculosis Inhibitors.. Cell Chemical Biology 2016, 23, 666-77, 10.1016/j.chembiol.2016.05.011.

93. Bharati Pandey; Sonam Grover; Jagdeep Kaur; Abhinav Grover; Analysis of mutations leading to para-aminosalicylic acid resistance in Mycobacterium tuberculosis.. Scientific Reports 2019, 9, 13617-15, 10.1038/s41598-019-48940-5.

94. Wenjing Wei; Huimin Yan; Jiao Zhao; Haicheng Li; Zhenyan Li; Huixin Guo; Xuezhi Wang; Ying Zhou; Xiaoli Zhang; Jincheng Zeng; Tao Chen; Lin Zhou; Multi-omics comparisons of p-aminosalicylic acid (PAS) resistance in folC mutated and un-mutated Mycobacterium tuberculosis strains.. Emerging Microbes \& Infections 2019, 8, 248-261, 10.1080/22221751.2019.1568179.

95. Navisha Dookie; Santhuri Rambaran; Nesri Padayatchi; Sharana Mahomed; Kogieleum Naidoo; Evolution of drug resistance in Mycobacterium tuberculosis: a review on the molecular determinants of resistance and implications for personalized care. Journal of Antimicrobial Chemotherapy 2018, 73, 1138-1151, 10.1093/jac/dkx506.

96. Dheda, K.; Gumbo, T.; Maartens, G.; Dooley, K.E.; Murray, M.; Furin, J.; Nardell, E.A.; Warren, R.M. On behalf of the Lancet Respiratory Medicine drug-resistant tuberculosis Commission group. The Lancet Respiratory Medicine Commission: 2019 update: Epidemiology, pathogenesis, transmission, diagnosis, and management of multidrug-resistant and incurable tuberculosis. Lancet Respir. Med. 2019, 7, 820-826.

97. Meehan, C.J.; Goig, G.A.; Kohl, T.A.; Verboven, L.; Dippenaar, A.; Ezewudo, M.; Farhat, M.R.; Guthrie, J.L.; Laukens, K.; Miotto, P.; 
et al.et al. Whole genome sequencing of Mycobacterium tuberculosis: current standards and open issues.. Nat. Rev. Microbiol. 2019, $17,533-545,10.1038 /$ s41579-019-0214-5.

98. World Health Organization. The Use of Next-Generation Sequencing Technologies for the Detection of Mutations Associated with Drug Resistance in Mycobacterium tuberculosis Complex: Technical Guide; World Health Organization: Geneva, Switzerland, 2018.

99. Pipeline Report 2019. Diagnostics. Treatment Action Group (TAG). Available online: https://www.treatmentactiongroup.org/wpcontent/uploads/2019/12/pipeline_tb_diagnotics_2019_db_final.pdf (accessed on 24 February 2020)

100. World Health Organization. Rapid Communication: Molecular Assays as Initial Test for the Diagnosis of Tuberculosis and Rifampicin Resistance. Available online: https://apps.who.int/iris/bitstream/handle/10665/330395/9789240000339-eng.pdf (accessed on 24 February 2020)

101. Markus Beutler; Sara Plesnik; Marina Mihalic; Laura Olbrich; Norbert Heinrich; Samuel Schumacher; Michael Lindner; Ina Koch; Wolfgang Grasse; Christoph Metzger-Boddien; Sabine Hofmann-Thiel; Harald Hoffmann; A pre-clinical validation plan to evaluate analytical sensitivities of molecular diagnostics such as BD MAX MDR-TB, Xpert MTB/Rif Ultra and FluoroType MTB. PLOS ONE 2020, 15, e0227215, 10.1371/journal.pone.0227215.

102. Pilar Ruiz; Manuel Causse; Manuel Vaquero; Juan Bautista Gutierrez; Manuel Casal; Evaluation of a new automated Abbott RealTime MTB RIF/INH assay for qualitative detection of rifampicin/isoniazid resistance in pulmonary and extra-pulmonary clinical samples of Mycobacterium tuberculosis. Infection and Drug Resistance 2017, 10, 463-467, 10.2147/IDR.S147272.

103. Margaretha De Vos; Brigitta Derendinger; Tania Dolby; John Simpson; Paul D. Van Helden; John E. Rice; Lawrence J. Wangh; Grant Theron; R. M. Warren; Diagnostic Accuracy and Utility of FluoroType MTBDR, a New Molecular Assay for Multidrug-Resistant Tuberculosis. Journal of Clinical Microbiology 2018, 56, JCM.00531-18, 10.1128/jcm.00531-18.

104. Jayshree Advani; Renu Verma; Oishi Chatterjee; Praveen Kumar Pachouri; Prashant Upadhyay; Rajesh Singh; Jitendra Yadav; Farah Naaz; Raju Ravikumar; Shashidhar Buggi; et al.Mrutyunjay SuarUmesh D. GuptaAkhilesh PandeyDevendra S. ChauhanSrikanth TripathyHarsha GowdaT. S. Keshava Prasad Whole Genome Sequencing of Mycobacterium tuberculosis Clinical Isolates From India Reveals Genetic Heterogeneity and Region-Specific Variations That Might Affect Drug Susceptibility.. Frontiers in Microbiology 2019, 10, 309, 10.3389/fmicb.2019.00309.

105. Allix-Béguec, C.; Arandjelovic, I.; Bi, L.; Beckert, P.; Bonnet, M.; Bradley, P.; Cabibbe, A.M.; Cancino-Muñoz, I.; Caulfield, M.J.; Chaiprasert, A.; et al.et al. Prediction of Susceptibility to First-Line Tuberculosis Drugs by DNA Sequencing. New England Journal of Medicine 2018, 379, 1403-1415, 10.1056/nejmoa1800474.

\section{Keywords}

tuberculosis; Mycobacterium tuberculosis; rifampin; isoniazid; mechanisms of resistance; mutations; granulomas; caseum; cell envelope; dormancy 